\title{
Exact solitary wave and quasi-periodic wave solutions of the KdV-Sawada-Kotera-Ramani equation
}

\author{
Lijun Zhang ${ }^{1,2^{*}}$ and Chaudry Masood Khalique ${ }^{2}$
}

\section{"Correspondence:}

li-jun0608@163.com

'Department of Mathematics,

Zhejiang Sci-Tech University,

Hangzhou, Zhejiang 310018, China

${ }^{2}$ International Institute for

Symmetry Analysis and

Mathematical Modelling,

Department of Mathematical

Sciences, North-West University,

Mafikeng Campus, Mmabatho,

2735, South Africa

\section{Springer}

\begin{abstract}
In this paper we derive new exact solitary wave solutions and quasi-periodic traveling wave solutions of the KdV-Sawada-Kotera-Ramani equation by using a method which we introduce here for the first time. Firstly, we reduce the associated fourth-order nonlinear ordinary differential equation (ODE) into a solvable first-order nonlinear ODE to obtain new exact traveling wave solutions, including the solitary wave and periodic solutions. Furthermore, using the new method we derive the quasi-periodic wave solutions of this equation by assuming that the solutions of the corresponding higher-order ODE are the sum of the solutions of two solvable first-order nonlinear ODEs. This new method can be used to investigate the exact traveling wave solutions and quasi-periodic wave solutions of a general class of higher-order wave equations.
\end{abstract}

Keywords: nonlinear wave equations; traveling wave solutions; sub-equation method; quasi-periodic wave solutions

\section{Introduction}

In this paper we study the KdV-Sawada-Kotera-Ramani equation [1-16]

$$
u_{t}+a\left(3 u^{2}+u_{x x}\right)_{x}+b\left(15 u^{3}+15 u u_{x x}+u_{x x x x}\right)_{x}=0
$$

which was used to theoretically study the resonances of solitons in a one-dimensional space by Hirota and Ito [13]. Equation (1.1) is reduced to the KdV equation when $b=0$ and to the Sawada-Kotera equation when $a=0$; thus, it is a linear combination of the KdV equation and the Sawada-Kotera equation. The existence of conservation laws for this equation was proved by Konno [14]. Some traveling wave solutions were derived in [16] by the $\left(G^{\prime} / G\right)$-expansion method. In [15], the traveling wave solutions of (1.1) were studied by using the generalized auxiliary equation method. Unfortunately, too many undetermined coefficients were involved in this method and some conditions on these coefficients were ignored, and thus some wrong results were given in [15], which can be checked by Maple.

We aim to investigate the traveling wave solutions of the KdV-Sawada-Kotera-Ramani equation (1.1) in the form $u(x, t)=y(\xi)=y(x-c t)$, where $c$ is the wave speed. Under the traveling wave coordinates, (1.1) is transformed to the nonlinear ordinary differential equation of the independent variable $\xi$. By integrating the transformed ODE once with respect

(c) 2015 Zhang and Khalique. This article is distributed under the terms of the Creative Commons Attribution 4.0 International License (http://creativecommons.org/licenses/by/4.0/), which permits unrestricted use, distribution, and reproduction in any medium, provided you give appropriate credit to the original author(s) and the source, provide a link to the Creative Commons license, and indicate if changes were made. 
to $\xi$, we obtain

$$
\frac{d^{4} y}{d \xi^{4}}+\left(15 y+\frac{a}{b}\right) \frac{d^{2} y}{d \xi^{2}}+15 y^{3}+\frac{3 a}{b} y^{2}-\frac{c}{b} y+g=0
$$

where $g$ is a constant of integration. Clearly, $u(x, t)=y(\xi)=y(x-c t)$ is a traveling wave solution of (1.1) if and only if $y(\xi)$ satisfies (1.2) with the wave speed $c$ and any arbitrary constant $g$. Since the reduced ODE (1.2) is a fourth-order nonlinear equation which is equivalent to a four-dimensional system, it is very difficult to investigate (1.2) from the point of view of dynamical systems.

There are two classes of solitary waves, namely, embedded solitons and gas solitons that have been studied by many researches in the fields of nonlinear optics and water wave theory $[2,17-20]$. In fact, soliton solutions are typically presented by homoclinic solutions to saddle-center equilibrium and saddle-saddle equilibrium, respectively, of the associated ODEs which describe traveling waves of the model PDEs. By using the method of dynamical systems and Congrove's results [21], Li and Zhang [1] investigated the exact explicit gap soliton, embedded soliton, periodic, and quasi-periodic wave solutions of the KdV-Sawada-Kotera-Ramani equation.

Notice that (1.2) contains $\frac{d^{4} y}{d \xi^{4}}, \frac{d^{2} y}{d \xi^{2}}$, and a polynomial of $y$. Clearly, the general form of (1.2) is given by

$$
\frac{d^{4} y}{d \xi^{4}}+(A y+B) \frac{d^{2} y}{d \xi^{2}}+D y^{3}+E y^{2}+F y+G=0
$$

which is actually a special case of the equation

$$
\frac{d^{4} y}{d \xi^{4}}+(A y+B) \frac{d^{2} y}{d \xi^{2}}+C\left(\frac{d y}{d \xi}\right)^{2}+D y^{3}+E y^{2}+F y+G=0
$$

with $C=0$. In fact, there exist a lot of nonlinear wave equations and some time-fractional nonlinear wave equations whose corresponding ODEs or their reduced ODEs are special cases of (1.4). See for example [1, 3, 9, 22, 23].

Recently by using the sub-equation method and dynamical system analysis, the bifurcation and exact solutions of (1.4) were studied in [22, 23]. Following the idea and the results in [22], the bifurcations and exact traveling wave solutions to the KdV-Sawada-KoteraRamani equation are obtained in Section 1 . The sub-equation method has been proposed and well applied in studying the exact solutions of nonlinear differential equations [6-11]. The main idea of the sub-equation method is to assume that the solutions to higher-order ODEs are polynomials of some functions satisfying a simpler equation. However, we observe that a family of solutions to (1.3) can be the sum of two solutions to a second-order ODE which can be reduced to a first-order nonlinear ODE. By using the exact solutions and bifurcations of this sub-equation which was derived in [23], some new traveling wave solutions and quasi-periodic traveling wave solutions of the KdV-Sawada-Kotera-Ramani equation are derived in Section 2. 


\section{A family of exact traveling wave solutions of the KdV- Sawada-Kotera-Ramani equation}

\subsection{Preliminaries}

Equation (1.3) is a special case of (1.4), which is (1.6) in [22] with $C=0$. Thus, from Theorem 2.1 and Theorem 2.2 in [22], we have the corresponding theorems regarding (1.3).

Theorem 2.1 Suppose that $D \leq 3 A^{2} / 40$. The function $y=y(\xi)$ solves the fourth-order differential (1.3) if it solves equation

$$
\left(\frac{d y}{d \xi}\right)^{2}=a_{3} y^{3}+a_{2} y^{2}+a_{1} y+a_{0}
$$

where

$$
\begin{aligned}
& a_{3}=\frac{-3 A \pm \sqrt{9 A^{2}-120 D}}{30}, \\
& a_{2}=-\frac{3 B a_{3}+2 E}{15 a_{3}+2 A}, \\
& a_{1}=-\frac{2\left(B a_{2}+a_{2}^{2}+F\right)}{9 a_{3}+A}, \\
& a_{0}=-\frac{B a_{1}+a_{1} a_{2}+2 G}{6 a_{3}} .
\end{aligned}
$$

Note that all the denominators in (2.2) are assumed to be nonzero. If the denominator of $a_{i}$ in (2.2) is zero, then $a_{i}$ can be arbitrary constant provided the numerator is also zero.

Theorem 2.2 Let $_{ \pm}=\frac{2 \Delta\left(-a_{2} \pm \sqrt{\Delta}\right)+3 a_{1} a_{2} a_{3}}{54 a_{3}^{2}}$ and $y_{e}^{ \pm}=\frac{-a_{2} \pm \sqrt{\Delta}}{3 a_{3}}$, where $\Delta=a_{2}^{2}-3 a_{1} a_{3}>0$, then the following conclusions hold:

(1) For $a_{0}=2 h_{+},(2.1)$ has a bounded solution approaching $y_{e}^{+}$as $\xi$ goes to infinity, which can be expressed as

$$
u=\frac{-a_{2}+\sqrt{\Delta}}{3 a_{3}}-\frac{\sqrt{\Delta}}{a_{3}} \operatorname{sech}^{2}\left[\frac{1}{2} \Delta^{\frac{1}{4}}\left(\xi-\xi_{0}\right)\right]
$$

a constant solution

$$
y=\frac{-a_{2}+\sqrt{\Delta}}{3 a_{3}},
$$

and an unbounded solution

$$
u=\frac{-a_{2}+\sqrt{\Delta}}{3 a_{3}}+\frac{\sqrt{\Delta}}{a_{3}} \operatorname{csch}^{2}\left[\frac{1}{2} \Delta^{\frac{1}{4}}\left(\xi-\xi_{0}\right)\right]
$$

where $\xi_{0}$ is an arbitrary constant.

(2) For $a_{0} \in\left(2 h_{-}, 2 h_{+}\right)$, if $a_{3}>0$, then for any $y_{3} \in\left(\frac{-a_{2}-2 \sqrt{\Delta}}{3 a_{3}}, \frac{-a_{2}-\sqrt{\Delta}}{3 a_{3}}\right)$,

$$
y=y_{3}-\frac{1}{2}\left(3 y_{3}+\frac{a_{2}}{a_{3}}+\sqrt{\Delta_{+}}\right) \operatorname{sn}^{2}\left(\Omega_{+}\left(\xi-\xi_{0}\right), k_{+}\right),
$$


is a family of smooth periodic solutions of (2.1). Here $k_{+}=2 \frac{\sqrt{3 y_{3}^{2}+2 \frac{a_{2}}{a_{3}} y_{3}+\frac{a_{1}}{a_{3}}}}{-3 y_{3}-\frac{a_{2}}{a_{3}}+\sqrt{\Delta_{+}}}, \Omega_{+}=\frac{\sqrt{2}}{4} \times$ $\sqrt{-3 a_{3} y_{3}-a_{2}+a_{3} \sqrt{\Delta_{+}}}$and $\Delta_{+}=\left(\frac{a_{2}}{a_{3}}\right)^{2}-3 y_{3}^{2}-2 \frac{a_{2}}{a_{3}} y_{3}-4 \frac{a_{1}}{a_{3}}$.

If $a_{3}<0$, then, for any $y_{1} \in\left(\frac{-a_{2}-\sqrt{\Delta}}{3 a_{3}}, \frac{-a_{2}-2 \sqrt{\Delta}}{3 a_{3}}\right)$,

$$
y=y_{1}-\frac{1}{2}\left(3 y_{1}+\frac{a_{2}}{a_{3}}-\sqrt{\Delta_{-}}\right) \operatorname{sn}^{2}\left(\Omega_{-}\left(\xi-\xi_{0}\right), k_{-}\right)
$$

is a family of smooth periodic solutions of (2.1). Here $k_{-}=2 \frac{\sqrt{3 y_{1}^{2}+2 \frac{a_{2}}{a_{3}} y_{1}+\frac{a_{1}}{a_{3}}}}{3 y_{1}+\frac{a_{2}}{a_{3}}+\sqrt{\Delta_{-}}}, \Omega_{-}=\frac{\sqrt{2}}{4} \times$ $\sqrt{-3 a_{3} y_{1}-a_{2}-a_{3} \sqrt{\Delta_{-}}}$, and $\Delta_{-}=\left(\frac{a_{2}}{a_{3}}\right)^{2}-3 y_{1}^{2}-2 \frac{a_{2}}{a_{3}} y_{1}-4 \frac{a_{1}}{a_{3}}$.

(3) For $a_{0} \in\left(-\infty, 2 h_{-}\right] \cup\left(2 h_{+},+\infty\right)$, (2.1) has no non-trivial bounded solutions. When $a_{0}=2 h_{-}$, an unbounded solution is given by

$$
y=-\frac{a_{2}+\sqrt{\Delta}}{3 a_{3}}+\frac{\sqrt{\Delta}}{a_{3}} \sec ^{2}\left[\frac{1}{2} \Delta^{\frac{1}{4}}\left(\xi-\xi_{0}\right)\right]
$$

and a constant solution

$$
y=-\frac{a_{2}+\sqrt{\Delta}}{3 a_{3}}
$$

\subsection{A family of exact traveling wave solutions of (1.1) obtained from Theorems 2.1 and 2.2}

By letting $A=15, B=a / b, D=15, E=3 a / b, F=-c / b$, and $G=g$, (1.3) is reduced to (1.2). Clearly $D=15<3 A^{2} / 40$. Thus from Theorem 2.1 , we know that $y=y(\xi)$ solves (1.2) if it solves the first-order nonlinear ODE (2.1) with $a_{3}=-1, a_{2}=-a /(5 b), a_{1}=$ $\left(4 a^{2}+25 b c\right) /\left(75 b^{2}\right)$, and $a_{0}=\left(375 b^{3} g+8 a^{3}+50 a b c\right) /\left(1,125 b^{3}\right)$. Note that $g$ in $(1.2)$ is an arbitrary constant, so $a_{0}$ is also an arbitrary constant. Consequently, we obtain the solutions of (1.2) from Theorem 2.2 provided $a_{2}^{2}-3 a_{1} a_{3}=\left(a^{2}+5 b c\right) /\left(5 b^{2}\right)>0$, i.e., $c>-a^{2} /(5 b)$ for $b>0$ or $c<-a^{2} /(5 b)$ for $b<0$.

Theorem 2.3 The KdV-Sawada-Kotera-Ramani equation (1.1) has the following traveling wave solutions with the wave speed $c$ satisfying $a^{2}+5 b c>0$ :

(1) It has a bounded solitary wave solution,

$$
y=-\frac{a}{15 b}-\frac{\sqrt{5\left(a^{2}+5 b c\right)}}{15|b|}+\frac{\sqrt{5\left(a^{2}+5 b c\right)}}{5|b|} \operatorname{sech}^{2}\left[\frac{1}{2}\left(\frac{a^{2}+5 b c}{5 b^{2}}\right)^{\frac{1}{4}}\left(x-c t-\xi_{0}\right)\right]
$$

where $\xi_{0}$ is an arbitrary constant.

$$
\begin{aligned}
& \text { (2) For any } y_{1} \in\left(-\frac{a}{15 b}+\frac{\sqrt{5\left(a^{2}+5 b c\right)}}{15|b|},-\frac{a}{15 b}+2 \frac{\sqrt{5\left(a^{2}+5 b c\right)}}{15|b|}\right) \\
& y=y_{1}-\frac{1}{2}\left(3 y_{1}+\frac{a}{5 b}-\sqrt{\Delta_{1}}\right) \operatorname{sn}^{2}\left(\Omega_{1}\left(x-c t-\xi_{0}\right), k_{1}\right)
\end{aligned}
$$

is a family of smooth periodic traveling wave solutions of the KdV-Sawada-Kotera-Ramani equation (1.1). Here $\Omega_{1}=\frac{\sqrt{2}}{4} \sqrt{3 y_{1}+\frac{a}{5 b}+\sqrt{\Delta_{1}}}, k_{1}=\frac{\sqrt{12 y_{1}^{2}+8 \frac{a}{5 b} y_{1}-4 \frac{4 a^{2}+25 b c}{75 b^{2}}}}{3 y_{3}+\frac{a}{5 b}+\sqrt{\Delta_{1}}}$, and $\Delta_{1}=-3 y_{1}^{2}-$ $\frac{2 a}{5 b} y_{1}+\frac{3 a^{2} c+16 a^{2}+100 b c}{75 b^{2}}$. 
(3) It has two classes of unbounded solutions,

$$
y=-\frac{a}{15 b}-\frac{\sqrt{5\left(a^{2}+5 b c\right)}}{15|b|}-\frac{\sqrt{5\left(a^{2}+5 b c\right)}}{5|b|} \operatorname{csch}^{2}\left[\frac{1}{2}\left(\frac{a^{2}+5 b c}{5 b^{2}}\right)^{\frac{1}{4}}\left(x-c t-\xi_{0}\right)\right]
$$

and

$$
y=-\frac{a}{15 b}+\frac{\sqrt{5\left(a^{2}+5 b c\right)}}{15|b|}-\frac{\sqrt{5\left(a^{2}+5 b c\right)}}{5|b|} \sec ^{2}\left[\frac{1}{2}\left(\frac{a^{2}+5 b c}{5 b^{2}}\right)^{\frac{1}{4}}\left(x-c t-\xi_{0}\right)\right] .
$$

\section{Exact quasi-periodic traveling wave solutions of the KdV-}

\section{Sawada-Kotera-Ramani equation}

In this section, we obtain a new family of exact traveling wave solutions of the KdVSawada-Kotera-Ramani equation (1.1), which includes the quasi-periodic solutions.

\subsection{A new family of exact solutions and quasi-periodic solutions of (1.3)}

Let $y=U+V$, then (1.3) is reduced to

$$
\begin{aligned}
& \left(\frac{d^{4} U}{d \xi^{4}}+(A U+B) \frac{d^{2} U}{d \xi^{2}}+D U^{3}+E U^{2}+F_{1} U+g_{1}\right) \\
& +\left(\frac{d^{4} V}{d \xi^{4}}+(A V+B) \frac{d^{2} V}{d \xi^{2}}+D V^{3}+E V^{2}+F_{2} V+g_{2}\right) \\
& +V\left(A \frac{d^{2} U}{d \xi^{2}}+3 D U^{2}+E U+\left(F-F_{2}\right)\right) \\
& +U\left(A \frac{d^{2} V}{d \xi^{2}}+3 D V^{2}+E V+\left(F-F_{1}\right)\right)=0
\end{aligned}
$$

where $F_{1}$ and $F_{2}$ are some constants to be determined later, $g_{1}$ and $g_{2}$ are arbitrary constants. Clearly, $y=U+V$ solves (1.3) if there exist some values of $F_{1}$ and $F_{2}$ such that $U$ and $V$ satisfy

$$
\left\{\begin{array}{l}
A \frac{d^{2} U}{d \xi^{2}}+3 D U^{2}+E U+\left(F-F_{2}\right)=0 \\
\frac{d^{4} U}{d \xi^{4}}+(A U+B) \frac{d^{2} U}{d \xi^{2}}+D U^{3}+E U^{2}+F_{1} U+g_{1}=0
\end{array}\right.
$$

and

$$
\left\{\begin{array}{l}
A \frac{d^{2} V}{d \xi^{2}}+3 D V^{2}+E V+\left(F-F_{1}\right)=0 \\
\frac{d^{4} V}{d \xi^{4}}+(A V+B) \frac{d^{2} V}{d \xi^{2}}+D V^{3}+E V^{2}+F_{2} V+g_{2}=0
\end{array}\right.
$$

respectively.

Suppose $U$ satisfies the first equation of system (3.2). Multiplying it by $\frac{d U}{d \xi}$ and integrating once, we obtain

$$
\left(\frac{d U}{d \xi}\right)^{2}=-\frac{2 D}{A} U^{3}-\frac{E}{A} U^{2}-2 \frac{F-F_{2}}{A} U+G_{1}
$$


where $G_{1}$ is an integral constant. By letting $a_{3}=-2 D / A, a_{2}=-E / A$, and $a_{1}=-2\left(F-F_{2}\right) / A$, from Theorem 2.1, we know that $U$ solves system (3.2) if and only if

$$
\begin{aligned}
& -\frac{2 D}{A}=\frac{-3 A \pm \sqrt{9 A^{2}-120 D}}{30}, \\
& -\frac{E}{A}=-\frac{3 B a_{3}+2 E}{15 a_{3}+2 A}, \\
& -2 \frac{F-F_{2}}{A}=-\frac{2\left(B a_{2}+a_{2}^{2}+F_{1}\right)}{9 a_{3}+A} .
\end{aligned}
$$

Solving the first equation of system (3.5) for $D$ gives $D=A^{2} / 15$ and thus $a_{3}=-2 A / 15$. By substituting the value of $a_{3}$ into the second equation and solving for $E$, we have $E=A B / 5$ and $a_{2}=-B / 5$. Then substituting the values of $a_{3}$ and $a_{2}$ into the third equation of (3.5) gives $F-F_{2}+5 F_{1}=4 B^{2} / 5$. In the same way, from system (3.3), we can obtain $F-F_{1}+5 F_{2}=$ $4 B^{2} / 5$. Consequently, we can determine the two undetermined constants $F_{1}$ and $F_{2}$ as $F_{1}=F_{2}=B^{2} / 5-F / 4$. We thus have the following theorem.

Theorem 3.1 Suppose that the coefficients of (1.3) satisfy $D=A^{2} / 15$ and $E=A B / 15$. Then $y(\xi)=U(\xi)+V(\xi)$ solves (1.3) if $U$ and $V$ satisfy equation

$$
\frac{d^{2} \phi}{d \xi^{2}}=-\frac{1}{5} A \phi^{2}-\frac{1}{5} B \phi-\left(\frac{5}{4 A} F-\frac{1}{5 A} B^{2}\right) .
$$

Obviously, (3.6) is equivalent to the first-order ODE

$$
\left(\frac{d \phi}{d \xi}\right)^{2}=-\frac{2}{15} A \phi^{3}-\frac{1}{5} B \phi^{2}-\left(\frac{5}{8 A} F-\frac{1}{10 A} B^{2}\right) \phi+G
$$

if $d \phi / d \xi \neq 0$ except for the case when $\phi=\left(-B \pm \sqrt{5 B^{2}-25 F}\right) /(2 A)$. Here $G$ is an arbitrary integral constant. From (3.7), we can obtain the solutions of (1.3).

\subsection{Exact solutions and quasi-periodic solutions of the KdV-Sawada-Kotera-Ramani equation (1.1)}

It is easy to see that the associated ODE (1.2) of the KdV-Sawada-Kotera-Ramani equation is the fourth-order $\operatorname{ODE}(1.3)$ with $A=15, B=a / b, D=15, E=3 a / b, F=-c / b$, and $G=g$. Clearly, the coefficients of (1.2) satisfy the conditions of Theorem 3.1, that is, $D=A^{2} / 15$ and $E=A B / 5$. Thus Theorem 3.1 implies that (1.2) admits the solutions $y=U+V$, where $U$ and $V$ are determined by

$$
\frac{d^{2} \phi}{d \xi^{2}}=-3 \phi^{2}-\frac{a}{5 b} \phi-\frac{4 a^{2}+25 b c}{75 b^{2}}
$$

or

$$
\left(\frac{d \phi}{d \xi}\right)^{2}=-2 \phi^{3}-\frac{a}{5 b} \phi^{2}-\frac{4 a^{2}+25 b c}{150 b^{2}} \phi+G
$$

Note that $G$ is an arbitrary constant, $U$ and $V$ are not constants except $-a \pm \sqrt{5 a^{2}+25 b c}$ / $(30 b)$. Consequently, we know that when the wave speed $c$ satisfies $a^{2}+5 b c>0$, i.e., 
$c>-a^{2} /(5 b)$ for $b>0$ or $c<-a^{2} /(5 b)$ for $b<0$, (3.8) admits the following six classes of solutions for different values of $G_{1}$ and $G_{2}$ :

$$
\begin{aligned}
\phi_{1}(\xi)= & \frac{-a+\sqrt{5 a^{2}+25 b c}}{30 b} ; \\
\phi_{2}(\xi)= & \frac{-a-\sqrt{5 a^{2}+25 b c}}{30 b} ; \\
\phi_{3}(\xi)= & -\frac{\operatorname{sgn}(b) a+\sqrt{5 a^{2}+25 b c}}{30|b|} \\
& +\frac{\sqrt{5 a^{2}+25 b c}}{10|b|} \operatorname{sech}^{2}\left[\frac{1}{2}\left(\frac{a^{2}+5 b c}{5 b^{2}}\right)^{\frac{1}{4}}\left(\xi-\xi_{0}\right)\right] ; \\
\phi_{4}(\xi)= & -\frac{\operatorname{sgn}(b) a+\sqrt{5 a^{2}+25 b c}}{30|b|} \\
& -\frac{\sqrt{5 a^{2}+25 b c}}{10|b|} \operatorname{csch}^{2}\left[\frac{1}{2}\left(\frac{a^{2}+5 b c}{5 b^{2}}\right)^{\frac{1}{4}}\left(\xi-\xi_{0}\right)\right] ; \\
\phi_{5}(\xi)= & -\frac{\operatorname{sgn}(b) a-\sqrt{5 a^{2}+25 b c}}{30|b|} \\
& -\frac{\sqrt{5 a^{2}+25 b c}}{10|b|} \sec ^{2}\left[\frac{1}{2}\left(\frac{a^{2}+5 b c}{5 b^{2}}\right)^{\frac{1}{4}}\left(\xi-\xi_{0}\right)\right] ;
\end{aligned}
$$

For any $\theta \in\left(\frac{-\operatorname{sgn}(b) a+\sqrt{5 a^{2}+25 b c}}{30|b|}, \frac{-\operatorname{sgn}(b) a+2 \sqrt{5 a^{2}+25 b c}}{30|b|}\right)$,

$$
\phi_{6}(\xi, \theta)=\theta-\frac{1}{2}\left(3 \theta+\frac{a}{10 b}-\sqrt{\Delta_{2}}\right) \operatorname{sn}^{2}\left(\Omega_{2}\left(\xi-\xi_{0}\right), k_{2}\right)
$$

where $\Omega_{2}(\theta, c)=\frac{\sqrt{2}}{4} \sqrt{6 \theta+\frac{a}{5 b}+2 \sqrt{\Delta_{2}}}, k_{2}(\theta, c)=\frac{\sqrt{12 \theta^{2}+4 \frac{a}{5 b} \theta-\frac{4 a^{2}+25 b c}{75 b^{2}}}}{3 \theta+\frac{a}{10 b}+\sqrt{\Delta_{2}}}$, and $\Delta_{2}(\theta, c)=-3 \theta^{2}-$ $\frac{a}{5 b} \theta+\frac{19 a^{2}+100 b c}{300 b^{2}}$.

According to the above analysis and Theorem 3.1, we obtain the solutions of (1.2) and consequently the exact traveling wave solutions of the KdV-Sawada-Kotera-Ramani equation (1.1). We have the following theorem.

Theorem 3.2 The KdV-Sawada-Kotera-Ramani equation (1.1) admits the following traveling wave solutions: $y_{i j}(x, t)=\phi_{i}(\xi, c)+\phi_{j}(\xi, c), i, j \in\{1,2,3,4,5,6\}$, with $\xi=x-c t$. Here $\phi_{i} s$ are determined by (3.10)-(3.15), respectively, and the wave speed c satisfies $a^{2}+5 b c>0$.

In fact, from Theorem 3.2, we can obtain the following three classes of solitary wave solutions; two families of periodic wave solutions, a family of quasi-periodic wave solutions, and some unbounded solutions.

(1) For any constant $c$ satisfying $a^{2}+5 b c>0$,

$$
\begin{aligned}
y_{33}(x, t)= & 2 \phi_{3}(\xi)=-\frac{\operatorname{sgn}(b) a+\sqrt{5 a^{2}+25 b c}}{15|b|} \\
& +\frac{\sqrt{5 a^{2}+25 b c}}{5|b|} \operatorname{sech}^{2}\left[\frac{1}{2}\left(\frac{a^{2}+5 b c}{5 b^{2}}\right)^{\frac{1}{4}}\left(x-c t-\xi_{0}\right)\right],
\end{aligned}
$$




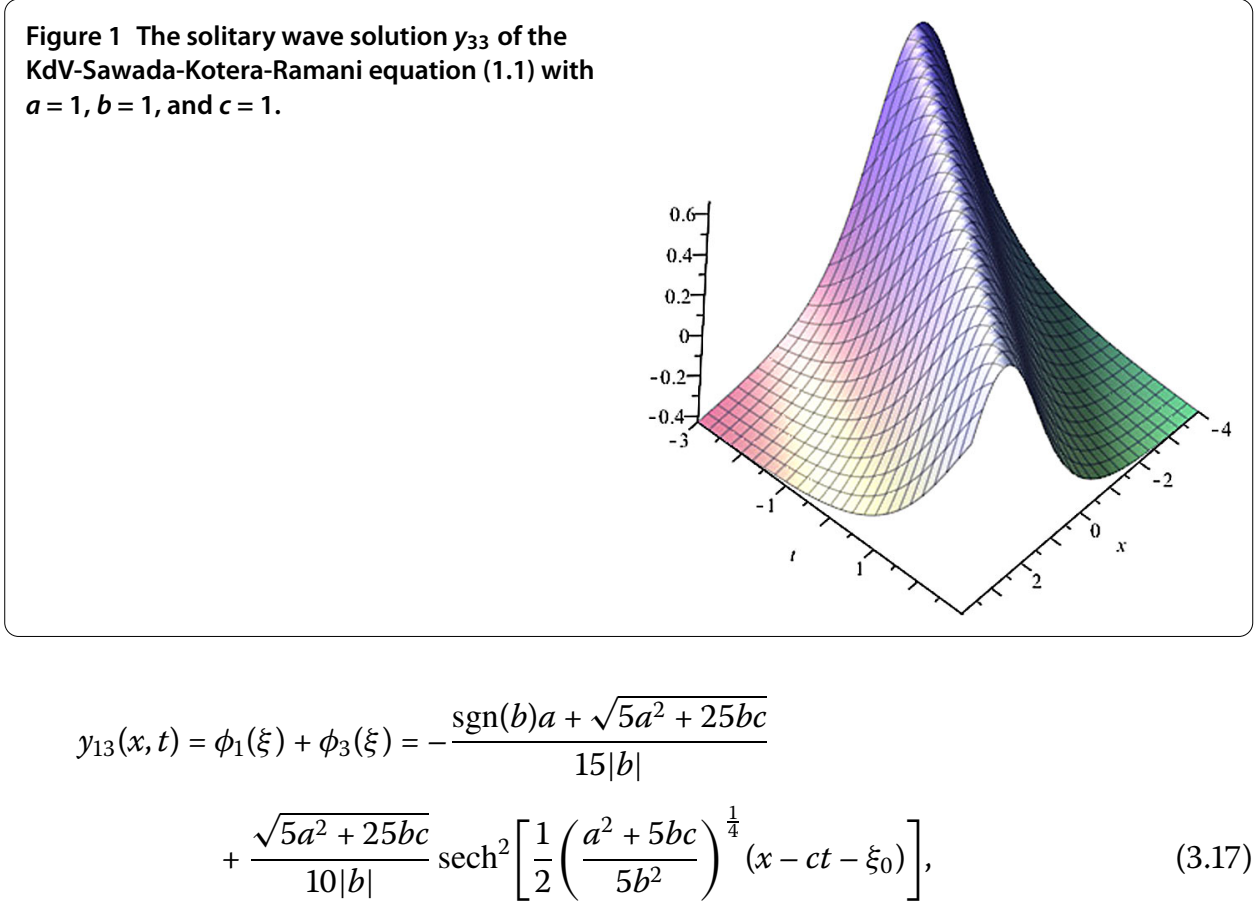

and

$$
\begin{aligned}
y_{23}(x, t) & =\phi_{2}(\xi)+\phi_{3}(\xi) \\
& =-\frac{a}{15 b}+\frac{\sqrt{5 a^{2}+25 b c}}{10|b|} \operatorname{sech}^{2}\left[\frac{1}{2}\left(\frac{a^{2}+5 b c}{5 b^{2}}\right)^{\frac{1}{4}}\left(x-c t-\xi_{0}\right)\right]
\end{aligned}
$$

are three classes of solitary wave solutions of the KdV-Sawada-Kotera-Ramani equation. The phase portrait of solution (3.16) with $a=1, b=1$, and $c=1$ is shown in Figure 1 . Note that solution (3.16) is the same as solution (2.10) obtained earlier in Section 2, solution (3.17) is a new solitary wave solution and solution (3.18) is the solitary wave solution (76) obtained in [24].

(2) For any $\theta \in\left(\frac{-\operatorname{sgn}(b) a+\sqrt{5 a^{2}+25 b c}}{30|b|}, \frac{-\operatorname{sgn}(b) a+2 \sqrt{5 a^{2}+25 b c}}{30|b|}\right)$, and any constant $c$ satisfying $a^{2}+$ $5 b c>0$,

$$
\begin{aligned}
y_{16}(x, t)= & \phi_{1}(\xi)+\phi_{6}(\xi, \theta)=\frac{-a+\sqrt{5 a^{2}+25 b c}}{30 b}+\theta \\
& -\frac{1}{2}\left(3 \theta+\frac{a}{10 b}-\sqrt{\Delta_{2}}\right) \operatorname{sn}^{2}\left(\Omega_{2}\left(x-c t-\xi_{0}\right), k_{2}\right)
\end{aligned}
$$

and

$$
\begin{aligned}
y_{26}(x, t)= & \phi_{2}(\xi)+\phi_{6}(\xi, \theta)=\frac{-a-\sqrt{5 a^{2}+25 b c}}{30 b}+\theta \\
& -\frac{1}{2}\left(3 \theta+\frac{a}{10 b}-\sqrt{\Delta_{2}}\right) \operatorname{sn}^{2}\left(\Omega_{2}\left(x-c t-\xi_{0}\right), k_{2}\right)
\end{aligned}
$$

are two families of periodic wave solutions of the KdV-Sawada-Kotera-Ramani equation. Here $\Omega_{2}=\frac{\sqrt{2}}{4} \sqrt{6 \theta+\frac{a}{5 b}+2 \sqrt{\Delta_{2}}}, k_{2}=\frac{\sqrt{12 \theta^{2}+4 \frac{a}{5 b} \theta-\frac{4 a^{2}+25 b c}{75 b^{2}}}}{3 \theta+\frac{a}{10 b}+\sqrt{\Delta_{2}}}$, and $\Delta_{2}=-3 \theta^{2}-\frac{a}{5 b} \theta+\frac{19 a^{2}+100 b c}{300 b^{2}}$. 


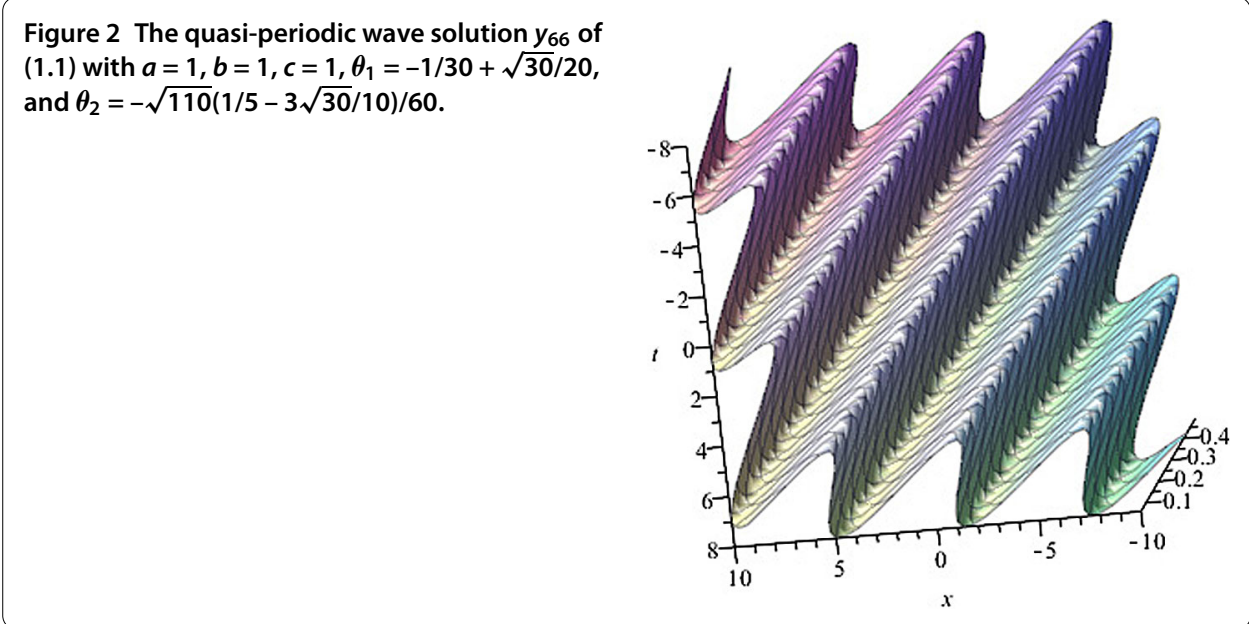

(3) For any constant $c$ satisfying $a^{2}+5 b c>0$ and two arbitrary constants $\theta_{1}$ and $\theta_{2}$ satisfying $\theta_{i} \in\left(\frac{-\operatorname{sgn}(b) a+\sqrt{5 a^{2}+25 b c}}{30|b|}, \frac{-\operatorname{sgn}(b) a+2 \sqrt{5 a^{2}+25 b c}}{30|b|}\right), i=1$, 2, we have

$$
\begin{aligned}
y_{66}(x, t)= & \phi_{6}\left(\xi, \theta_{1}\right)+\phi_{6}\left(\xi, \theta_{2}\right) \\
= & \theta_{1}-\frac{1}{2}\left(3 \theta_{1}+\frac{a}{10 b}-\sqrt{\Delta_{2}\left(\theta_{1}\right)}\right) \operatorname{sn}^{2}\left(\Omega_{2}\left(\theta_{1}\right)\left(x-c t-\xi_{0}\right), k_{2}\left(\theta_{1}\right)\right) \\
& +\theta_{2}-\frac{1}{2}\left(3 \theta_{2}+\frac{a}{10 b}-\sqrt{\Delta_{2}\left(\theta_{2}\right)}\right) \operatorname{sn}^{2}\left(\Omega_{2}\left(\theta_{2}\right)\left(x-c t-\xi_{0}\right), k_{2}\left(\theta_{2}\right)\right),
\end{aligned}
$$

where $\Omega_{2}\left(\theta_{i}\right)=\frac{\sqrt{2}}{4} \sqrt{6 \theta_{i}+\frac{a}{5 b}+2 \sqrt{\Delta_{2}\left(\theta_{i}\right)}}, k_{2}\left(\theta_{i}\right)=\frac{\sqrt{12 \theta_{i}^{2}+4 \frac{a}{5 b} \theta_{i}-\frac{4 a^{2}+25 b c}{75 b^{2}}}}{3 \theta_{i}+\frac{a}{10 b}+\sqrt{\Delta_{2}\left(\theta_{i}\right)}}$, and $\Delta_{2}\left(\theta_{i}\right)=-3 \theta_{i}^{2}-$ $\frac{a}{5 b} \theta_{i}+\frac{19 a^{2}+100 b c}{300 b^{2}}, i=1,2$. Note that (3.21) with $\theta_{1}=\theta_{2}$ is a class of periodic traveling wave solutions (2.11), which were obtained in Section 2. However, (3.21) is a family of quasiperiodic traveling wave solutions when $\theta_{1} \neq \theta_{2}$ and $\Omega_{2}\left(\theta_{1}\right) / \Omega_{2}\left(\theta_{2}\right)$ is irrational.

The phase portrait of (3.21) with $a=1, b=1, c=1, \theta_{1}=-1 / 30+\sqrt{30} / 20$, and $\theta_{2}=$ $-\sqrt{110}(1 / 5-3 \sqrt{30} / 10) / 60$ is shown in Figure 2.

(4) For any $\theta \in\left(\frac{-\operatorname{sgn}(b) a+\sqrt{5 a^{2}+25 b c}}{30|b|}, \frac{-\operatorname{sgn}(b) a+2 \sqrt{5 a^{2}+25 b c}}{30|b|}\right)$, and any constant $c$ satisfying $a^{2}+$ $5 b c>0$,

$$
\begin{aligned}
y_{36}(x, t)= & \phi_{3}(\xi)+\phi_{6}(\xi, \theta)=-\frac{\operatorname{sgn}(b) a+\sqrt{5 a^{2}+25 b c}}{30|b|}+\theta \\
& +\frac{\sqrt{5 a^{2}+25 b c}}{10|b|} \operatorname{sech}^{2}\left[\frac{1}{2}\left(\frac{a^{2}+5 b c}{5 b^{2}}\right)^{\frac{1}{4}}\left(\xi-\xi_{0}\right)\right] \\
& -\frac{1}{2}\left(3 \theta+\frac{a}{10 b}-\sqrt{\Delta_{2}}\right) \operatorname{sn}^{2}\left(\Omega_{2}\left(x-c t-\xi_{0}\right), k_{2}\right)
\end{aligned}
$$

is a family of bounded wave solutions of the KdV-Sawada-Kotera-Ramani equation (see Figure 3). Here $\Omega_{2}=\frac{\sqrt{2}}{4} \sqrt{6 \theta+\frac{a}{5 b}+2 \sqrt{\Delta_{2}}}, k_{2}=\frac{\sqrt{12 \theta^{2}+4 \frac{a}{5 b} \theta-\frac{4 a^{2}+25 b c}{75 b^{2}}}}{3 \theta+\frac{a}{10 b}+\sqrt{\Delta_{2}}}$, and $\Delta_{2}=-3 \theta^{2}-\frac{a}{5 b} \theta+$ $\frac{19 a^{2}+100 b c}{300 b^{2}}$. 


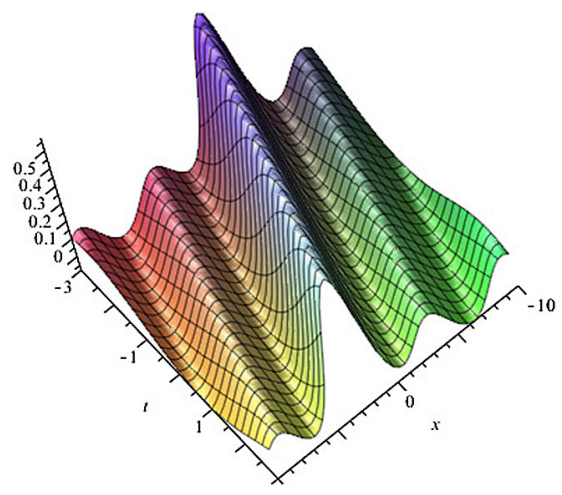

(a)

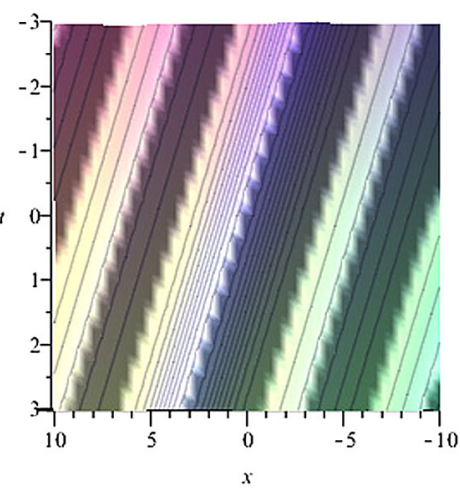

(b)

Figure 3 The bounded wave solution $y_{36}$ of (1.1) with $a=1, b=1$, and $c=1$. (a) Three-dimensional portrait; (b) overhead view with contour plot.

(5) For any constant $c$ satisfying $a^{2}+5 b c>0$,

$$
\begin{aligned}
& y_{14}(x, t)=\phi_{1}(\xi)+\phi_{4}(\xi)=-\frac{\operatorname{sgn}(b) a-\sqrt{5 a^{2}+25 b c}}{15|b|} \\
& -\frac{\sqrt{5 a^{2}+25 b c}}{10|b|} \operatorname{csch}^{2}\left[\frac{1}{2}\left(\frac{a^{2}+5 b c}{5 b^{2}}\right)^{\frac{1}{4}}\left(x-c t-\xi_{0}\right)\right] ; \\
& y_{24}(x, t)=\phi_{1}(\xi)+\phi_{4}(\xi)=-\frac{\operatorname{sgn}(b) a+\sqrt{5 a^{2}+25 b c}}{15|b|} \\
& -\frac{\sqrt{5 a^{2}+25 b c}}{10|b|} \operatorname{csch}^{2}\left[\frac{1}{2}\left(\frac{a^{2}+5 b c}{5 b^{2}}\right)^{\frac{1}{4}}\left(x-c t-\xi_{0}\right)\right] ; \\
& y_{15}(x, t)=\phi_{1}(\xi)+\phi_{5}(\xi)=-\frac{\operatorname{sgn}(b) a-\sqrt{5 a^{2}+25 b c}}{15|b|} \\
& -\frac{\sqrt{5 a^{2}+25 b c}}{10|b|} \sec ^{2}\left[\frac{1}{2}\left(\frac{a^{2}+5 b c}{5 b^{2}}\right)^{\frac{1}{4}}\left(x-c t-\xi_{0}\right)\right] \\
& y_{25}(x, t)=\phi_{2}(\xi)+\phi_{5}(\xi)=-\frac{\operatorname{sgn}(b) a}{15|b|} \\
& -\frac{\sqrt{5 a^{2}+25 b c}}{10|b|} \sec ^{2}\left[\frac{1}{2}\left(\frac{a^{2}+5 b c}{5 b^{2}}\right)^{\frac{1}{4}}\left(x-c t-\xi_{0}\right)\right] ; \\
& y_{44}(x, t)=2 \phi_{4}(\xi)=-\frac{\operatorname{sgn}(b) a+\sqrt{5 a^{2}+25 b c}}{15|b|} \\
& -\frac{\sqrt{5 a^{2}+25 b c}}{5|b|} \operatorname{csch}^{2}\left[\frac{1}{2}\left(\frac{a^{2}+5 b c}{5 b^{2}}\right)^{\frac{1}{4}}\left(\xi-\xi_{0}\right)\right] ; \\
& y_{55}(x, t)=2 \phi_{5}(\xi)=-\frac{\operatorname{sgn}(b) a-\sqrt{5 a^{2}+25 b c}}{15|b|} \\
& -\frac{\sqrt{5 a^{2}+25 b c}}{5|b|} \sec ^{2}\left[\frac{1}{2}\left(\frac{a^{2}+5 b c}{5 b^{2}}\right)^{\frac{1}{4}}\left(\xi-\xi_{0}\right)\right] ;
\end{aligned}
$$




$$
\begin{aligned}
y_{45}(x, t)= & \phi_{4}(\xi)+\phi_{5}(\xi)=-\frac{\operatorname{sgn}(b) a}{15|b|} \\
& -\frac{\sqrt{5 a^{2}+25 b c}}{10|b|}\left(\operatorname{csch}^{2}+\sec ^{2}\right)\left[\frac{1}{2}\left(\frac{a^{2}+5 b c}{5 b^{2}}\right)^{\frac{1}{4}}\left(\xi-\xi_{0}\right)\right]
\end{aligned}
$$

are unbounded solutions of the KdV-Sawada-Kotera-Ramani equation. Note that solutions (3.24) and (3.25) are the solutions (2.12) and (2.13), respectively.

\section{Conclusion and discussion}

In this paper, we studied the exact traveling wave solutions to the KdV-Sawada-KoteraRamani equation (1.1) via the sub-equation in the form $(d y / d \xi)^{2}=a_{3} y^{3}+a_{2} y^{2}+a_{1} y+a_{0}$. The sub-equation of similar form, namely $(d y / d \xi)^{2}=P_{m}(y)$, where $P_{m}(y)$ is a polynomial of $y$, has been applied to investigate some nonlinear wave equations [4-11]. In all these papers, the solutions to the original equations are usually the polynomial functions of the solutions to the sub-equations. However, by using the new method introduced in this paper (the sum of two solutions to sub-equations), we obtained many new exact traveling wave solutions to the KdV-Sawada-Kotera-Ramani equation (1.1). Especially, some quasiperiodic wave solutions were derived by using this new method. Furthermore, we obtained a very general class of exact solutions of the KdV-Sawada-Kotera-Ramani equation (1.1), which included the solitary wave solutions, periodic and quasi-periodic traveling wave solutions and some unbounded traveling solutions as well. Our results are more general than those obtained previously in the literature. For example, the solutions (12) and (14) in [16] actually can be rewritten as our solutions (3.16) and (3.27), respectively. Unfortunately, (18) and (20) in [16] do not satisfy (1.1) and hence are not the solutions of (1.1).

It is well known that not only the exact solutions but also the bifurcations of the dynamical systems can be investigated by using the dynamical system theorem [24, 25]. The planar dynamical system method has been well applied in studying the traveling wave solutions of various nonlinear wave solutions [1, 22, 23, 26-31]. However, it is usually very difficult to study the systems in a higher-dimensional space unless they can be reduced to a two-dimensional space. Normally, the higher-order differential equations can be reduced to a lower-dimensional space provided that their first integrals can be derived $[1$, 21,28 ]. Unfortunately, it is usually intractable to derive their first integrals. In this paper, we reduced the higher-order ODE into planar dynamical system by finding its lower-order sub-equation. Whether there are any other kinds of sub-equations possessed by this class of equations is still an open problem.

The method proposed in this paper can be applied to other nonlinear wave equations, especially to higher-order nonlinear wave equations. This might pave the way to the study of the exact traveling wave solutions of higher-order nonlinear wave equations. However, whether and how this method can be used to investigate the multiple-wave solutions of higher-order nonlinear wave equations will be the topic of our future study. 


\section{Acknowledgements}

L Zhang thanks the North-West University for the post-doctoral fellowship. This work is supported by the Nature Science Foundation of China (No. 11101371, No. 11422214).

Received: 30 December 2014 Accepted: 17 May 2015 Published online: 26 June 2015

\section{References}

1. Li, JB, Zhang, Y: Homoclinic manifolds, center manifolds and exact solutions of four-dimensional traveling wave systems for two classes of nonlinear wave equations. Int. J. Bifurc. Chaos 21(2), 527-543 (2011)

2. Yang, J: Dynamics of embedded solitons in the extended Korteweg-de Vries equations. Stud. Appl. Math. 106, 337-365 (2001)

3. Inc, M, Kilic, B: Classification of traveling wave solutions for time-fractional fifth-order KdV-like equation. Waves Random Complex Media 24(2), 393-403 (2014)

4. Inc, M: Some special structures for the generalized nonlinear Schrödinger equation with nonlinear dispersion. Waves Random Complex Media 23(2), 77-88 (2013)

5. Kilic, B, Inc, M: The first integral method for the time fractional Kaup-Boussinesq system with time dependent coefficient. Appl. Math. Comput. 254, 70-74 (2015)

6. Ma, WX, Lee, JH: A transformed rational function method and exact solutions to the $(3+1)$-dimensional Jimbo-Miwa equation. Chaos Solitons Fractals 42(3), 1356-1363 (2009)

7. Zhang, S, Xia, T: A generalized new auxiliary equation method and its applications to nonlinear partial differential equations. Phys. Lett. A 363, 356-360 (2007)

8. Li, H, Wang, KM, Li, JB: Exact traveling wave solutions for the Benjamin-Bona-Mahony equation by improved Fan sub-equation method. Appl. Math. Model. 37, 7644-7652 (2013)

9. Parkes, EJ, Duffy, BR, Abbott, PC: The Jacobi elliptic-function method for finding periodic-wave solutions to nonlinear evolution equations. Phys. Lett. A 295, 280-286 (2002)

10. Feng, DH, Li, KZ: Exact traveling wave solutions for a generalized Hirota-Satsuma coupled KdV equation by Fan sub-equation method. Phys. Lett. A 375, 2201-2210 (2011)

11. Fan, E: Uniformly constructing a series of explicit exact solutions to nonlinear equations in mathematical physics. Chaos Solitons Fractals 16, 819-839 (2003)

12. Fan, E: Supersymmetric KdV-Sawada-Kotera-Ramani equation and its quasi-periodic wave solutions. Phys. Lett. A 374 744-749 (2010)

13. Hirota, R, Ito, M: Resonance of solitons in one dimension. J. Phys. Soc. Jpn. 52(3), 744-748 (1983)

14. Konno, K: Conservation laws of modified Sawada-Kotera equation in complex plane. J. Phys. Soc. Jpn. 61, 51-54 (1992)

15. Zhang, J, Zhang, J, Bo, L: Abundant travelling wave solutions for KdV-Sawada-Kotera equation with symbolic computation. Appl. Math. Comput. 203, 233-237 (2008)

16. Qin, Z, Mu, G, Ma, H: G'/G-Expansion method for the fifth-order forms of KdV-Sawada-Kotera equation. Appl. Math. Comput. 222, 29-33 (2013)

17. Champneys, AR, Groves, MD, Woods, PD: A global characterization of gap solitary wave solutions to a coupled KdV system. Phys. Lett. A 271, 178-190 (2000)

18. Champneys, AR, Malomed, BA, Yang, J, Kaup, DJ: Embedded solitons: solitary wave in resonance with the linear spectrum. Physica D 152, 340-354 (2001)

19. Yang, J, Malomed, BA, Kaup, DJ, Champneys, AR: Embedded solitons: a new type of solitary wave. Math. Comput. Simul. 56, 585-600 (2001)

20. Yagasaki, K, Wagenknecht, T: Detection of symmetric homoclinic orbits to saddle-centres in reversible systems. Physica D 214, 169-181 (2006)

21. Cosgrove, MC: Higher-order Painlevé equations in the polynomial class I. Bureau symbol P2. Stud. Appl. Math. 104, $1-65(2000)$

22. Zhang, LJ, Khalique, CM: Exact Solitary wave and periodic wave solutions of a class of higher-order nonlinear wave equations. (submitted)

23. Zhang, LJ, Khalique, CM: Exact solitary wave and periodic wave solutions of the Kaup-Kuperschmidt equation. J. Appl. Anal. Comput. 5(3), 485-495 (2015)

24. Guckenheimer, J, Holmes, P: Dynamical Systems and Bifurcations of Vector Fields. Springer, New York (1983)

25. Chow, SN, Hale, JK: Method of Bifurcation Theory. Springer, New York (1981)

26. Li, JB: Singular Traveling Wave Equations: Bifurcations and Exact Solutions. Science Press, Beijing (2013)

27. Li, JB, Chen, GR: Bifurcations of traveling wave solutions for four classes of nonlinear wave equations. Int. J. Bifurc. Chaos 15(12), 3973-3998 (2005)

28. Li, JB, Qiao, ZJ: Explicit solutions of the Kaup-Kuperschmidt equation through the dynamical system approach. J. Appl. Anal. Comput. 1(2), 243-250 (2011)

29. Zhang, LJ, Chen, LQ, Huo, XW: The effects of horizontal singular straight line in a generalized nonlinear Klein-Gordon model equation. Nonlinear Dyn. 72(4), 789-801 (2013)

30. Rui, WG: Different kinds of exact solutions with two-loop character of the two-component short pulse equations of the first kind. Commun. Nonlinear Sci. Numer. Simul. 18, 2667-2678 (2013)

31. Chen, AY, Wen, SQ, Huang, WT: Existence and orbital stability of periodic wave solutions for the nonlinear Schrödinger equation. J. Appl. Anal. Comput. 2(2), 137-148 (2012) 